\title{
State-dependent retention induced by postacquisition exposure to pentobarbital or shock stress in rats
}

\author{
RICK RICHARDSON, DAVID C. RICCIO, and JOHN H. STEELE \\ Kent State University, Kent, Ohio
}

\begin{abstract}
Although state-dependent retention (SDR) has been the focus of considerable research in recent years, there has been little work demonstrating this phenomenon when the treatment is administered after learning. In the present study, the effectiveness of two different treatments, pentobarbital and shock-induced stress, in producing postacquisition SDR in rats was examined. In all experiments, water-deprived rats were exposed to a novel flavor, apple juice, on each of 2 days. Subjects that remember their initial exposure to this substance should show an increase in consumption on their second exposure (i.e., an attenuation of neophobia). It was found that subjects exposed to pentobarbital (Experiment 1) or shock-induced stress (Experiment 2) immediately after their initial exposure did not increase their intake on the subsequent exposure unless they were reexposed to pentobarbital (Experiment 1) or shock-induced stress (Experiment 2b) shortly prior to that exposure. These results clearly show that this "attenuation of neophobia" paradigm can be used to investigate postacquisition SDR.
\end{abstract}

Although both experimentally induced amnesia and state-dependent retention (SDR) produce impairment of memory, historically they have been usually treated as separate phenomena. However, it is becoming increasingly clear that considerable commonality exists between these two sources of memory loss. For instance, in the familiar $2 \times 2$ design used in much SDR work, it is found that subjects exposed to drug prior to both training and testing exhibit no performance deficit (e.g., Ebner, Richardson, \& Riccio, 1981; see Overton, 1978, for a review). Although early studies of induced amnesia typically focused on the comparison between groups treated (or not) at training, when treatment at testing is also considered (in effect, the other half of the SDR factorial design), the similarity of the amnesia and SDR phenomena becomes more apparent: Reexposing subjects to the amnestic treatment prior to testing eliminates or greatly attenuates the retention deficit (see Riccio \& Richardson, 1984, for a review). For example, electroconvulsive shock (ECS), hypothermia, and hyperthermia are all potent amnestic treatments when given either after acquisition (i.e., "retrograde amnesia"; Mactutus, Ferek, \& Riccio, 1980; Miller \& Marlin, 1979; Misanin, Vonheyn, Bartelt, Boulden, \& Hinderliter, 1979; Riccio, Hodges \& Randall, 1968) or before acquisition (i.e., "anterograde amnesia"; Ahlers, 1984; Gardner, Glick, \& Jarvik, 1972; Richardson, Riccio, \& Morilak, 1983), yet in both paradigms the

This research was supported in part by NIMH Grant MH37535 to D. C. Riccio. During preparation of this report, R. Richardson was supported by NRSA MH09179 from NIMH. Requests for reprints can be sent to either D. C. Riccio, at Department of Psychology, Kent State University, Kent, OH 44242, or R. Richardson, who is now at the Psychology Department, Princeton University, Princeton, NJ 08544. memory loss is readily reversed by exposing subjects to the amnestic agent prior to test (Ahlers, 1984; Gardner et al., 1972; Hinderliter, Webster, \& Riccio, 1976; Miller \& Marlin, 1979; Richardson, Guanowsky, Ahlers, \& Riccio, 1984). Thus, in many respects it would appear that whether "forgetting" in a given situation is referred to as SDR or experimentally induced amnesia might be largely a matter of preference in terminology.

Despite the general similarity of these phenomena, a potentially important difference does exist with respect to the effects on retention produced by postacquisition administration of the two treatments. Amnestic agents such as ECS or hypothermia are capable of disrupting memory whether they are applied prior to or shortly following training. Although earlier studies focused on the retrograde memory loss produced by traumatic insult such as ECS or hypothermia (e.g., Madsen \& McGaugh, 1961; Riccio et al., 1968), more recent research has shown that "surprising"' posttrial events (Kremer, 1979; Wagner, Rudy, \& Whitlow, 1973) or administration of hormones such as ACTH (e.g., Gold \& van Buskirk, 1976) can also impair subsequent retention. In contrast, it appears that memory loss with SDR agents may be limited to the anterograde condition in which learning occurs in the drugged state. Thus, there is little evidence that posttrial administration of pentobarbital, a traditional statedependent agent, influences memory retrieval, although administration of this drug prior to training readily produces a strong state-dependent retention effect (Overton, 1964, 1978). It should be noted that a state-dependentlike effect has been reported when pentobarbital was administered following passive avoidance training (Chute \& Wright, 1973), but that finding seems to be an isolated one. Given the delays over which retrograde amnesia can 
be obtained (e.g., Riccio et al., 1968), and the relatively rapid onset of physiological changes produced by pentobarbital, it seems doubtful that these negative findings can be attributed to an unduly long functional interval between training and drug action.

Since traditional "amnestic agents" are highly effective when presented after training, and the vast majority of experimentally induced amnesia studies have examined retrograde amnesia (for reviews, see Miller \& Marlin, 1979; Riccio \& Richardson, 1984), the virtual absence of demonstrations of postacquisition effects of SDR agents is puzzling. It could be that agents such as ECS and disruption of body temperature act on underlying memory mechanisms so differently from typical SDR-producing agents that no similarity of outcomes should be expected. On the other hand, given the other apparent parallels among these treatments, perhaps the lack of commonality in postacquisition effects reflects quantitative rather than qualitative differences. If pentobarbital were a "weaker" agent along a continuum of severity, then memory based upon punishment training (widely used in this area of research) may be relatively resistant to the drug's postacquisition influence. This interpretation suggests that a task involving less potent reinforcement (what Strupp \& Levitsky, 1983, have termed "advantageous" learning) might be more sensitive to retroactive effects of pentobarbital.

In this respect, one task that might be suitable takes advantage of the fact that the neophobic responses of rats to a novel taste can be attenuated by prior exposure to the taste. However, if this initial sampling episode is forgotten, then rats should treat the flavor as though it were still novel (i.e., exhibit neophobia) on the subsequent exposure. Using this attenuation-of-neophobia paradigm, Burešova and Bureš (1981) demonstrated that deep-body cooling following novel consumption of apple juice produced retrograde amnesia for the flavor experience. Subsequently, our laboratory (Morilak, Orndoff, Riccio, $\&$ Richardson, 1983) showed that the attenuation of neophobia provided a sensitive indication of anterograde treatments. Rats that received a relatively low dose $(10 \mathrm{mg} / \mathrm{kg})$ of pentobarbital before their initial apple juice exposure were more neophobic when tested without the drug than were either of the two same-state control groups (drug-drug; no drug-no drug). Furthermore, a similar SDR effect was obtained when a stress state, induced by administering several brief but unpredictable and inescapable shocks, was the anterograde manipulation rather than pentobarbital. Thus, in the current study, the effects of administering pentobarbital or shock-induced stress after an initial exposure to apple juice were examined by measuring subsequent attenuation of neophobia.

\section{EXPERIMENT 1}

In the first experiment, we employed the "attenuation of neophobia" paradigm to detect a retrograde influence of pentobarbital. Following a brief exposure to a novel taste, rats were injected with pentobarbital or saline. Two days later, the animals were reexposed to the original taste substance. Shortly prior to this reexposure, subjects were injected with either the same agent (pentobarbital or saline) that they had received postacquisition, or the other agent. Subjects that remembered the initial exposure to the taste should increase consumption of this substance (i.e., exhibit an attenuation of neophobia), whereas subjects failing to remember the initial exposure should not increase their intakes. Thus, if pentobarbital was effective in producing postacquisition SDR in this paradigm, consumption of apple juice should be less in the drugsaline condition than in same-state conditions.

\section{Method}

Subjects. Forty-eight male Sprague-Dawley-derived rats (purchased from the Holtzman Co.) served as subjects. The animals were between 90 and 150 days of age at the beginning of the experiment. All subjects had been previously trained to fear the black compartment of a black/white shuttlebox in earlier experiments not related to the present study. These subjects had also either received injections of ACTH, dexamethasone, and/or saline or been subjected to hypothermia (deep-body cooling) in the previous studies. Subjects from these previous treatment conditions were assigned randomly to groups in the present experiment. Animals were individually housed throughout this experiment in wire-mesh cages and maintained on a 15:9-h light:dark cycle (lights on at $0730 \mathrm{~h}$ ). Food and water were available ad lib unless stated otherwise.

Procedure. The animals were handled for approximately $3 \mathrm{~min}$ a day for 2 days. Following handling on the 2 nd day, all subjects were water deprived. Twenty-four hours later, each subject was weighed and then transported, in its home cage, to a small testing room where it received an initial exposure to apple juice. After being weighed, each subject had its food removed to insure that the apple juice flavor was not "masked" by eating during the brief exposure period. This was done at training and testing in all experiments. During juice presentation, a graduated cylinder (Fischer Scientific) containing $11 \mathrm{ml}$ of unsweetened apple juice (White House brand, purchased from a local grocery) was attached to the home cage and the animal was allowed to drink for $5 \mathrm{~min}$. The graduated cylinder was then removed, and the subject was immediately injected, intraperitoneally, with either sodium pentobarbital (Nembutal, $12.5 \mathrm{mg} / \mathrm{kg}$ in a volume of $1 \mathrm{ml} / \mathrm{kg}$ ) or an equivalent volume of $0.9 \%$ physiological saline. The subject was then returned to the colony, where the water bottle and food were returned 1 to $2 \mathrm{~h}$ later. In this and all subsequent experiments, amounts of apple juice consumed were measured (to nearest $.2 \mathrm{ml}$ ) by an experimenter blind to subjects' group assignments.

Water bottles were again removed $24 \mathrm{~h}$ following the initial juice exposure. Subjects were randomly assigned to one of four treatment groups ( $n=12$ per group), which were derived by administer ing the two agents (saline and pentobarbital) in all possible combinations at training and test. Prior to their second exposure to the apple juice, the animals were weighed again and administered their respective dose of pentobarbital (Pento-Pento and Sal-Pento groups) or an equal volume of saline (Pento-Sal and Sal-Sal groups). (The first term of the two-word group designation refers to the subjects state at training, the second represents the condition at testing.) Following a 15-min delay, the subjects were transported to the same small testing room used for the initial juice presentation and given a 5 -min presentation of apple juice.

\section{Results}

A one-way ANOVA revealed no significant difference on initial intake among the four experimental groups 
$[F(3,44)<1]$. To assess retention of information about the initial exposure to apple juice, a ratio [Day 2 intake/(Day $1+$ Day 2 intake)] was used in which decreased neophobia (retention) was indicated by ratio scores exceeding .50. In this way, individual differences in absolute intake were controlled. These data are depicted in Figure 1. A two-way ANOVA on the ratio data revealed no main effect of treatment at training $[F(1,44)$ $=2.25, p>.10]$ or testing $[F(1,44)=1.07, p>.10]$. However, a significant interaction was found $[F(1,44)=$ 11.24, $p<.01]$. Subsequent Newman-Keuls tests $(p<.05)$ revealed that both same-state groups (Sal-Sal and Pento-Pento) increased their consumption of apple juice significantly more than the postacquisition drug group (Pento-Sal), although not significantly more than the other mismatched group (Sal-Pento).

\section{Discussion}

These data indicate that immediate postacquisition administration of pentobarbital can impair retention of a novel flavor, as reflected in the persistence of neophobic behavior. However, retention is normal if rats are returned to the drug state at testing (Pento-Pento), that is, consumption of the flavor is comparable to that of nondrugged controls (Sal-Sal).

Although pentobarbital may affect responding in a variety of ways, it is important to recognize that the overall pattern of outcomes largely precludes interpretations based on nonmemorial processes such as acquired taste aversion. For example, drinking in the Pento-Sal group at testing would also be depressed if the drug produced a conditioned taste aversion, but this interpretation is rendered very unlikely in view of the increased consumption of subjects in the Pento-Pento group, which also received the drug after initial taste exposure. Furthermore, it should be noted that subjects in the Pento-Sal condition consumed nearly the same amount of apple juice on both days (i.e., their ratio was near.5). If administering this dose of pentobarbital immediately after the juice exposure produced

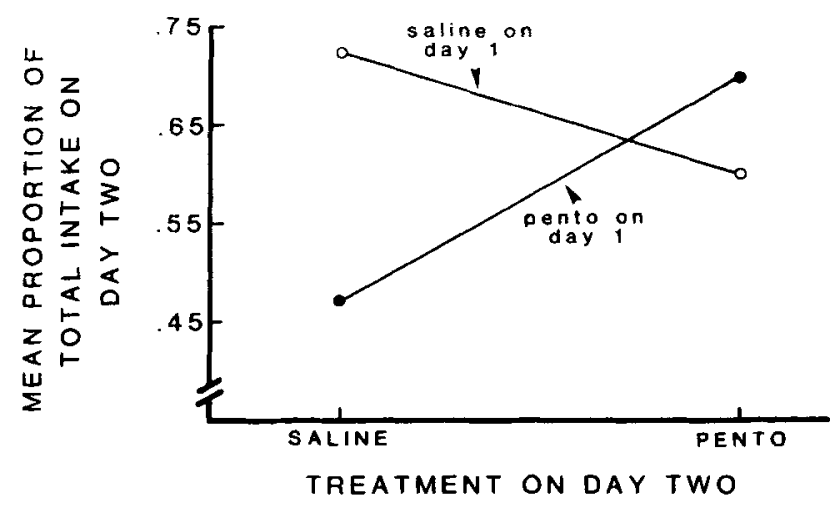

Figure 1. Mean proportion of total liquid intake consumed on the second of 2 days of exposure to apple juice for rats in Experiment 1. Treatment given on Day 1 occurred immediately after the exposure; that given on Day 2 occurred prior to the apple juice exposure. a conditioned taste aversion, then these subjects should have consumed less apple juice on Day 2 than on Day 1 . The pattern of results, then, seems quite consistent with memorial interpretations of other SDR research (cf. Spear, 1978). Furthermore, the asymmetrical outcome, in which the drug given only at testing fails to disrupt performance, is quite common in more traditional SDR-type studies.

That induction of a drug state immediately following exposure to a stimulus can impair later retention (in the absence of the drug) provides another point of similarity with the effects of traditional amnestic agents such as ECS or hypothermia. At the same time, an important difference may exist in that the learning task employed here did not involve a potent reinforcer, whereas ECS and hypothermia are routinely found to be effective in disrupting memory for episodes involving strong negative reinforcers, that is, punishment training.

As noted earlier, previous evidence of a postacquisition SDR effect has been scant. However, following completion of this manuscript, a study on the effects of postacquisition alcohol administration on memory in human subjects came to our attention. In addition to finding the traditional SDR when alcohol was given prior to training, Lowe (1983) reported that subjects who ingested alcohol immediately following a "map learning" task showed impaired retention if tested in the sober state. However, the impairment was eliminated if the subjects were intoxicated prior to testing. Conversely, intoxication prior to testing disrupted retention if alcohol had not been administered following training. Although Lowe's study obtained a symmetrical dissociation, the aspect of major relevance here is that administering the drug after the learning episode resulted in an SDR effect.

\section{EXPERIMENT 2A}

Experiment 1 provides evidence that the drug state induced by pentobarbital can, indeed, influence postacquisition information processing, at least of the type involved in habituation of a neophobic response. But we have previously suggested that the effect of endogenous bodily states on memory remains a relatively neglected, but important, aspect of SDR research (Ebner et al., 1981). There is evidence that circadian rhythms (Holloway, 1978; Holloway \& Wansley, 1973; Stroebel, 1967), shock stress (Morilak et al., 1983), and even mood states in humans (Bower, 1981) can act in a state-dependent manner. However, neither the naturally occurring changes associated with pregnancy or estrus nor the hormonal mimicking of estrus in ovariectomized rats appear to be dissociative (Ebner et al., 1981; Morilak et al., 1983), although administration of supraphysiological levels of progesterone will produce state dependency (Ebner et al., 1981; Stewart, Krebs, \& Kaczender, 1967). Although the temporal characteristics of some endogenous conditions preclude their use in a retrograde paradigm, induction of a stress reaction can be achieved quite rapidly. Because the stress state associated with a series of unpredictable, inescap- 
able shocks administered prior to flavor exposure is sufficient to produce anterograde memory loss when subjects are tested in a nonstressed condition (Morilak et al., 1983), a similar procedure was employed here in the retroactive design. As in Experiment 1, subjects were tested for attenuation of neophobia in one of four conditions representing the combinations of postacquisition stress (or not) and pretest stress (or not).

A methodological complication introduced by the Pavlovian conditioning contingencies inherent even in noncontingent shock treatment is that, by holding apparatus exposure constant, there is the possibility of inadvertently reactivating a fear (stress) response. Thus, in the shock-no-shock group, returning the subjects to the shock compartment (without shock) prior to testing equates handling and apparatus exposure with that of the shockshock group, but raises the obvious possibility that some degree of mild stress is elicited by conditioned fear cues prior to testing. In order to control for and evaluate this potentially interesting outcome, we included groups that were not placed, prior to testing, in the apparatus where noncontingent footshock had been given.

\section{Method}

Subjects. Seventy-two naive Long-Evans-derived male rats from the Kent State University Psychology Department breeding colony served as subjects in this experiment. The subjects were between 34 and 50 days of age at the beginning of the study. Housing conditions were the same as in Experiment 1.

Procedure. Apple juice presentation was done as in Experiment 1. Some subjects received noncontingent footshock (NCFS) in a $20 \times 17.5 \times 20 \mathrm{~cm}$ unpainted wooden box with a clear Plexiglas hinged lid. The floor consisted of grids, $0.6 \mathrm{~cm}$ in diameter and spaced $1.2 \mathrm{~cm}$ apart. Shock from a matched-impedance source (Campbell \& Teghtsoonian, 1958) was delivered through a Foringer scrambler.

The animals were handled as in Experiment 1. After the 2nd day of handling, water bottles were removed. Twenty-four hours later, the food was removed from the cages and the subjects were individually transported to the same testing room used in Experiment 1, where a 5-min presentation of apple juice was given. Immediately following the apple juice presentation, half of the subjects were placed in the wooden box, where they received four noncontingent footshocks $(200 \mathrm{~V} / 1 \mathrm{sec})$ administered every $15 \mathrm{sec}$ over a 1-min period. The remaining animals received a $1-$ min exposure to the wooden box without shock. The animals were then returned to the colony, where water and food were returned 1 to $2 \mathrm{~h}$ later.

Twenty-four hours later, the subjects were water deprived again. On the following day, two groups of subjects were returned to the wooden box and shocked (Groups SH-SH, NSH-SH). The other two groups were returned to the wooden box but not given shock (Groups SH-NSH and NSH-NSH). The final two groups were not returned to the wooden box prior to this second exposure to apple juice (Group SH-NSH, no box; Group NSH-NSH, no box). The first symbol in each group designation refers to the treatment given after the first juice presentation; the second indicates the treatment given prior to the second juice exposure. "SH" stands for "shock" and "NSH" for "no shock." Those animals not exposed to the wooden box prior to the second juice presentation have "no box" following their group designation. Each group contained 12 subjects.

At testing, all subjects remained undisturbed in their home cages for 2 min prior to presentation of the apple juice. This brief delay was intended to permit the development of any internal "stress" state as well as to reduce the influence of shock-induced freezing affecting consumption of fluid. This was done in Experiment 2B as well.

\section{Results and Discussion}

A one-way ANOVA on the ratio scores [Day 1/(Day 1 + Day 2)] indicated no significant treatment effects $[F(5,66)=1.0]$. Thus, it would appear that the shockinduced stress after initial exposure to a novel flavor was not effective in producing state-dependent retention for that experience. However, inspection of the data from this experiment reveals that subjects given shock after the first exposure to apple juice and not exposed to shock or the apparatus cues associated with shock at the time of testing showed very little increase in consumption of apple juice (mean $=.53$; all other groups had mean ratios $\geq .58$ ). As this is the direction of effect predicted from the SDR viewpoint, we decided to replicate the experiment using a more intense shock-induced stress.

\section{EXPERIMENT 2B}

In Experiment 2B, the number of shocks was increased by a factor of 10 in order to determine if a more intense postacquisition treatment would yield a retrograde statedependent retention effect.

\section{Method}

Sixty-three naive Long-Evans-derived male rats from the Kent State University Psychology Department breeding colony were used as subjects in this study. The subjects ranged from 63 to 86 days of age at the beginning of the experiment. Housing conditions, drinking apparatus, and shock apparatus were identical to those used in Experiment 2A. Four subjects were replaced (each of these subjects had consumed $.2 \mathrm{ml}$, or less, of juice on the initial exposure).

The experimental design was identical to that employed in Experiment $2 \mathrm{~A}$ except for the following changes. Although the shock voltage remained at 200 , subjects in the shock groups received 40 shocks ( 1 every $15 \mathrm{sec}$ ) during a 10 -min exposure. Consequently, subjects receiving only exposure to the wooden box (without shock) were placed in the box for $10 \mathrm{~min}$ to control for exposure to the novel apparatus. The subjects that received no shock and no wooden box exposure prior to the second juice presentation simply stayed in their home cages for $12 \mathrm{~min}$ before the apple juice presentation.

\section{Results}

A one-way ANOVA on initial intakes indicated that the experimental groups did not differ on their initial intake of apple juice $[F(5,57)<1]$. To test for the SDR effects of postacquisition shock on retention of consumption of a novel fluid, a two-way ANOVA was initially performed on the ratio data of subjects comprising the typical $2 \times 2$ design (i.e., Groups SH-SH, NSH-NSH, SH-NSH, and NSH-SH). Because group sizes were not equal (all groups contained 10 or 11 subjects each), an unweighted means analysis was employed. This analysis revealed a significant main effect of treatment at training $[F(1,39)=6.49$, $p<.025]$ and at testing $[F(1,39)=10.48, p<.01]$, and an interaction of these two conditions $[F(1,39)=$ $114.93, p<.001]$. This interaction is seen clearly in Figure 2. 


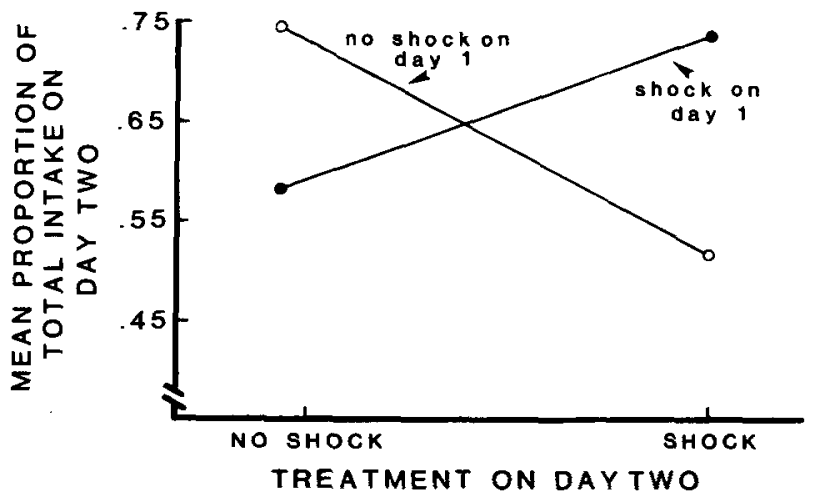

Figure 2. Mean proportion of total liquid intake consumed on the second of 2 days of exposure to apple juice for rats in Experiment 2B. Treatment given on Day 1 occurred immediately after the exposure; that given on Day 2 occurred prior to the apple juice exposure. Only the groups that comprised the traditional $2 \times 2$ design are shown.

To determine the effect on retention (if any) of exposure to the apparatus where shock had been given, a one-way ANOVA on the ratio data from all six groups of this study was performed (i.e., the four groups from the two-way ANOVA as well as Groups NSH-NSH, no box and SHNSH, no box). This analysis indicated a significant treatment effect $[F(5,57)=20.46, p<.01]$. Subsequent Newman-Keuls tests $(p<.05)$ indicated that the three same-state groups (i.e., SH-SH; NSH-NSH; NSH-NSH, no box) were all significantly different from the three mismatched-state groups (i.e., SH-NSH; SH-NSH, no box; NSH-SH). The three same-state groups did not differ from one another, nor did the three mismatched groups.
These data are depicted in Figure 3. This pattern of results reveals a symmetrical state-dependent retention effect.

\section{Discussion}

Experiment 2B provides clear evidence that the stress state elicited by noncontingent shocks can result in dissociated retention even when the shocks are administered following the acquisition trial. Although it could be proposed that shock served as a punishing function which suppressed later drinking, this interpretation encounters serious difficulty in explaining why a second aversive treatment should restore the punished response (Group $\mathrm{SH}-\mathrm{SH})$ rather than further reduce responding.

The difference in results obtained in Experiments $2 \mathrm{~A}$ and 2B could be attributed to the difference in animals' ages in the two experiments. A more likely interpretation of the difference in results, however, is that a relatively severe or prolonged shock stress is a necessary condition for the SDR effect. Independent support for this view is provided by a study which appeared after the present data were collected. In one of the conditions of a study that examined the effect on naloxone on posttrial administration of ethanol, Castellano and Pavone (1983) found that 30 or $60 \mathrm{~min}$ of immobilization stress following passive avoidance training in control mice produced a performance deficit at testing, whereas a 15-min treatment was without effect. The retrograde effects of immobilization stress (Castellano \& Pavone, 1983) and footshock (Experiment 2B of the present study) can be seen as complementary to the anterograde stress-induced SDR found by Morilak et al. (1983). However, it should be noted that the relatively mild shock regimen used in the

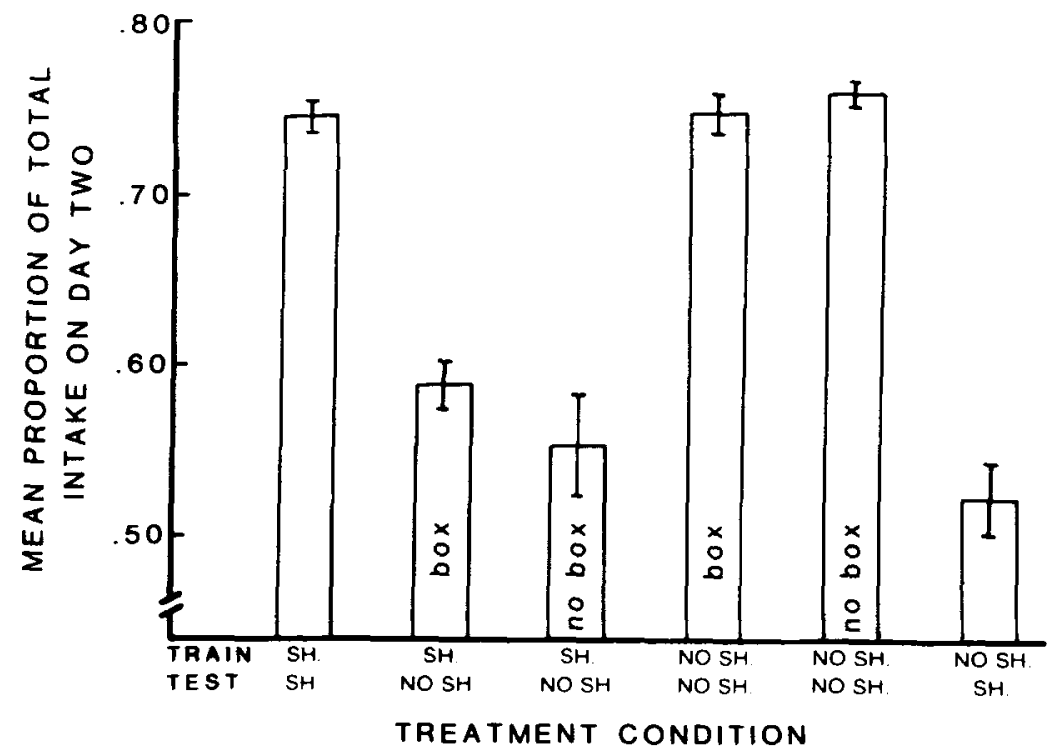

Figure 3. Mean proportion of total liquid intake consumed on the second of 2 days of exposure to apple juice for rats in Experiment 2B. Treatment given on Day 1 occurred immediately after the exposure; that given on Day 2 occurred prior to the apple juice exposure. All groups, including those not exposed to shock apparatus at all on Day 2, are shown. Standard errors of the means are indicated. 
anterograde paradigm of Morilak et al. failed to yield a postacquisition effect on memory (Experiment 2A).

Whether drugs or amnestic treatments are used to modulate memory, it is not uncommon to find that changing the state before or after acquisition is far more disruptive than changing the state at testing, that is, asymmetrical dissociation. For example, inducing hypothermia immediately after training produces a profound amnesia which, in turn, is reversible by re-administering hypothermia prior to testing. However, there is virtually no impairment of retention in the train-normal, test-hypothermia condition (Hinderliter et al., 1976). Experiment 1 of the present study provides another pertinent illustration of asymmetrical SDR. Thus, the finding of symmetrical state-dependent retention induced by postacquisition shock is somewhat surprising. The temptation to interpret the low intake in the NSH-SH group in terms of the disruptive effect of shock prior to testing is made untenable by the fact that the SH-SH group shows a significantly higher consumption of the fluid on the second exposure. Although the SH-SH group also had two exposures to the shock stress, it seems highly unlikely that any appreciable adaptation could have occurred following a single (first) exposure.

\section{GENERAL DISCUSSION}

The present findings provide evidence of posttrial statedependent-like effects with two quite different types of treatment: a pharmacological agent, pentobarbital, and an endogenous stress state produced by footshock. Thus, these data add to the evidence that information continues to be processed following an experience, and that the postacquisition internal environment can be an important source of encoding/retrieval cues. Although these treatments would not ordinarily be considered as amnestic agents, certain similarities seem inescapable. The most salient similarity is the reversal of the memory deficit by reexposure to the treatment prior to testing, a finding that has been obtained with amnestic agents such as ECS (see Miller \& Marlin, 1979) and hypothermia (see Riccio \& Richardson, 1984). Furthermore, Izquierdo and Dias (1983) have recently demonstrated a similar statedependent effect when either epinephrine or ACTH was injected following a punishment training trial. Subjects that received injections of these hormones after training would exhibit poor retention of the learned response unless the hormone was re-administered prior to test. It seems likely that the retrograde effect of shock on retention found in Experiment 2 also depends in part upon the role of ACTH and epinephrine, although in this case the stress hormones are endogenously released.

An interesting interpretive issue here is whether these findings represent a retrograde effect of treatment, as we have assumed, or whether the taste of apple juice might persist into the postacquisition period to produce an anterograde situation. That is, if the sensory stimulus were present during and after the drug administration, the paradigm would appear to be more properly classified as anterograde. This question can be seen as a variant of the warmly debated argument in the conditioned taste aversion (CTA) literature concerning peripheral versus more central representations (e.g., Bitterman, 1975; Revusky, 1971). Thus, some have argued that the acquisition of CTA with a long interval between the CS and UCS is simply attributable to a lingering taste that is then physically present or contiguous with illness, that is, delay rather than trace conditioning. A sensory-peripheral interpretation of the present data would suggest that although the altered state occurs following exposure to a flavor, the continued presence of the taste during drug exposure is tantamount to an anterograde treatment. Clearly, a retrograde interpretation also assumes some type of postacquisition processing, but it is the central nature of such a representation that makes the term "retrograde SDR" an appropriate concept. Introducing a delay of treatment does not resolve the question, since, without an independent index of the status of the sensory stimuli, the taste could be assumed to persist for any given interval. However, two considerations lead us to believe that the present phenomenon is based on a retrograde influence on a central representation. First, a variety of manipulations that should eliminate or reduce the persistence of a taste do not prevent acquisition of CTA with long delays of illness (e.g., Revusky, 1971). Second, using a sensory preconditioning paradigm to examine flavor-flavor associations, Lavin (1976) found no evidence that associations could be established with stimulus intervals of more than a few seconds. Presumably, if a taste remained after removal of the flavor stimulus, then it should be able to enter into associations with stimuli other than illness over long intervals.

The present findings may be pertinent to an issue concerning "distractors" and memory of flavors. Several studies have found that habituation of the neophobic response to a taste can be disrupted by presentation of a second, novel "distractor" flavor following exposure to the target stimulus (cf. Robertson \& Garrud, 1983). An important interpretation of this "disruption of attenuation of neophobia" effect is based upon Wagner's (1978) concept of limited stimulus processing capacity (cf. Atkinson \& Shiffrin, 1968). If the distractor flavor commands attention mechanisms, then processing of the target information is interrupted or limited, resulting in a weak representation in long-term memory. Thus, the organism fails to recognize the previously tasted flavor as "familiar" and treats it as novel (again). The present findings, by showing that drug and shock treatments following a novel taste also act as "distractors," may indicate that the flavor disruption in short-term memory (STM) is a special case of a more general phenomenon. We recognize, of course, that these similar outcomes could result from quite different, perhaps unrelated, mechanisms. But, if, for the sake of parsimony, it is assumed that some commonality exists between these phenomena, then it would appear that distractor events need not be 
limited to the sensory modality of the target stimulus. More importantly, perhaps the present data imply that disruption of STM is an inadequate explanation inasmuch as the memory was later recoverable. Thus, it would now seem to be of interest to examine the effects of presenting the distractor flavor after initial exposure and prior to testing-a design that seems not to have been used, reasonably enough, given an orientation toward examining STM or consolidation processes. Indeed, the conceptual scheme of postacquisition state dependency may prove to be applicable to other studies in which memory impairments are attributed to disruption of STM.

\section{REFERENCES}

AHLERS, S. T. (1984). Hyperthermia and anterograde amnesia. Unpublished MA thesis, Kent State University.

Atkinson, R. C., \& Shiffrin, R. M. (1968). Human memory: A proposed system and its control processes. In K. W. Spence \& J. T. Spence (Eds.), The psychology of learning and motivation (Vol. 2, pp. 89-105). New York: Academic Press.

BitTERman, M. E. (1975). The comparative analysis of learning. Science, 188, 699-709.

Bower, G. H. (1981). Mood and memory. American Psychologist, 36, 129-148.

BURESova, O., \& Bures, J. (1981). Threshold hypothermia disrupting acquisiton of conditioned taste aversion of neophobia in rats. Behavioral \& Neural Biology, 31, 274-282.

Campbell, B. A., \& Teghtsoonian, R. (1958). Electrical and behavioral effects of different types of shock stimuli on the rat. Journal of Comparative \& Physiological Psychology, 51, 185-192.

Castellano, C., \& Pavone, F. (1983). Naloxone-reversible effects of ethanol on passive avoidance behavior in mice. Physiological Psychology, 11, 291-295.

Chute, D. L., WRIGHT, D. C. (1973). Retrograde state-dependent learning. Science, 180, 878-879.

EBNER, D. L., RichardSON, R., \& Riccio, D. C. (1981). Ovarian hormones and retention of learned fear in rats. Behavioral \& Neural Biology, 33, 45-58.

GardNer, E. L., Glick, S. D., \& JARVIK, M. E. (1972). ECS dissociation of learning and one-way cross-dissociation with physostigmine and scopolamine. Physiology \& Behavior, 8, 11-15.

Gold, P. E., \& VAN BuskiRK, R. (1976). Enhancement and impairment of memory processes with posttrial injections of adrenocorticotrophic hormone. Behavioral Biology, 16, 387-400.

Hinderliter, C. F., Webster, T., \& Riccio, D. C. (1976). Amnesia induced by hypothermia as a function of treatment-test interval and recooling in rats. Animal Learning \& Behavior, 3, 257-263.

HollowaY, F. A. (1978). State-dependent retrieval based on time of day. In B. T. Ho, D. W. Richards III, \& D. L. Chute (Eds.), Drug discrimination and state dependent learning (pp. 319-343). New York: Academic Press.

Holloway, F. A., \& Wansley, R. A. (1973). Multiphasic retention deficits at periodic intervals after passive avoidance learning. Science, 180, 208-210.

IZquiERDO, I., \& DIAS, R. D. (1983). Memory as a state dependent phenomenon: Role of ACTH and epinephrine. Behavioral \& Neural Biology. 38, 144-149.

KREMER, E. F. (1979). Effect of posttrial episode on conditioning in compound conditioned stimuli. Journal of Experimental Psychology: Animal Behavior Processes, 5, 130-141.

LAvin, M. J. (1976). The establishment of flavor-flavor associations using a sensory preconditioning training procedure. Learning \& Motivation, 7, 173-183.

LowE, G. (1983). Alcohol and state-dependent learning. Substance \& Alcohol Actions/Misuse, 4, 273-282.

Mactutus, C. F., Ferek, J. M., \& Riccio, D. C. (1980). Amnesia induced by hyperthermia: An unusually profound, yet reversible memory loss. Behavioral \& Neural Biology, 30, 260-277.

MAdSEN, M. C., MCGAUGH, J. L. (1961). The effect of ECS on onetrial avoidance learning. Joumal of Comparative \& Physiological Psychology, 54, 522-523.

Miller, R. R., MARLIN, N. A. (1979). Amnesia following electroconvulsive shock. In J. F. Kihlstrom \& F. J. Evans (Eds.), Functional disorders of memory (pp. 143-178). Hillsdale, NJ: Erlbaum.

Misanin, J. R., Vonheyn, R. E., Bartelt, S. W., Boulden, W. L., \& Hinderliter, C. F. (1979). The effect of hyperthermia on memory in rats. Physiological Psychology, 7, 339-344.

Morilak, D. A., Orndoff, R. K., Riccio, D. C., \& Richardson, R. (1983). Persistence of flavor neophobia as an indicator of statedependent retention induced by pentobarbital, stress, and estrus. $B e$ havioral \& Neural Biology, 38, 47-60.

OVERTON, D. A. (1964). State-dependent or "dissociated" learning produced with pentobarbital. Journal of Comparative \& Physiological Psychology, 57, 3-12.

OVERTON, D. A. (1978). Major theories of state dependent learning. In B. T. Ho, D. W. Richards III, \& D. L. Chute (Eds.), Drug discrimination and state dependent learning (pp. 283-318). New York: Academic Press.

REvusky, S. (1971). The role of interference in association over a delay. In W. K. Honig \& P. H. R. James (Eds.), Animal memory (pp. 155-213). New York: Academic Press.

Riccio, D. C., Hodges, L. A., \& Randale, P. K. (1968). Retrograde amnesia produced by hypothermia in rats. Journal of Comparative \& Physiological Psychology, 66, 618-622.

Riccio, D. C., \& Richardson, R. (1984). The status of memory following experimentally induced amnesias: Gone, but not forgotten. Physiological Psychology, 12, 59-72.

Richardson, R., Guanowsky, V., Ahlers, S. T., \& Riccio, D. C. (1984). Role of body temperature in the onset of, and recovery from, hypothermia-induced anterograde amnesia. Physiological Psychology, 12, $125-132$.

Richardson, R., Riccio, D. C., \& Morilak, D. A. (1983). Anterograde memory loss induced by hypothermia in rats. Behavioral \& Neural Biology, 37, 76-88.

ROBERTSON, D., \& GARRUD, P. (1983). Variable processing of flavors in rat STM. Animal Learning \& Behavior, 11, 474-482.

SPEAR, N. E. (1978). The processing of memories: Forgetting and retention. Hillsdale, NJ: Erlbaum.

Stewart, J., Krebs, W. H., \& Kaczender, E. (1967). State-dependent learning produced with steroids. Nature (London), 216, 1223-1224.

STRUPP, B. J., \& LeviTSKY, D. A. (1983). Early brain insult and cognition: A comparison of malnutrition and hypothyroidism. Developmental Psychobiology, 16, 535-549.

STROEBEL, C. F. (1967). Behavioral aspects of circadian rhythms. In J. Zubin \& H. F. Hunt (Eds.), Comparative psychopathology (pp. 158-172). New York: Grune \& Stratton.

W AGNER, A. R. (1978). Expectancies and the priming of STM. In S. H. Hulse, H. Fowler, \& W. K. Honig (Eds.), Cognitive processes in animal behavior (pp. 177-209). Hillsdale, NJ: Erlbaum.

WAgner, A. R., RUDY, J. W., \& WhItLow, J. W., JR. (1973). Rehearsal in animal conditioning. Journal of Experimental Psychology Monograph, 97, 407-426.

(Manuscript received April 25, 1985; revision accepted for publication December 12, 1985.) 